\title{
Neural Networks Applied to Thermal Damage Classification in Grinding Process
}

\author{
Marcelo M. Spadotto, Paulo Roberto de Aguiar, Carlos C. P. Sousa, \\ Eduardo C. Bianchi \\ Sao Paulo State University - Unesp - Bauru Campus \\ Brazil
}

\section{Introduction}

Throughout the course of human progress from prehistoric times until now the technological world was characterized by the development and improvement of new methods to control the environment. One of the most obstacle to overcome in order to reach the complete automation of machining process within the integrated and flexible manufacturing systems is the development that can be named a non-human-assisted machining, that is, a process in which the moment for tool change, the tool change itself and the change of the grinding conditions no longer need the human being assistance. Thus, the development of monitoring and control systems in real time is of great importance. High temperatures in grinding process are the main source of thermal damages to the ground surface, which is a visible manifestation in steels known as grinding burn. Depending on the temperature reached in the grinding zone a burn degree on the part surface can be observed which is due to temper color from very thin oxide layers. One of the challenges found in the implementation of intelligent grinding process is the automatic detection of surface burn of the parts. Several systems of monitoring have been assessed by researchers in order to control the grinding process and guarantee the quality of the ground parts. However, monitoring techniques still fails in certain situations where the phenomenon changes are not completely obtained by the employed signals.

Several monitoring systems which use force or power and acoustic emission sensors have been assessed by researchers to control surface burn in grinding (Aguiar et al.,2002; Aguiar et al., 1998; Kwak \& Song, 2001; Wang et al., 2001; Kwak \& Ha, 2004; Dotto et al., 2006; Aguiar et al., 2006a; Aguiar et al., 2006b). However, those techniques still need improvements where the phenomenon variations are not entirely acquired by the signals used.

High temperatures in grinding are the main source of thermal damages to the ground surface. A visible manifestation of this damage in steels is grinding burn - a discoloration of the ground surface often visible directly to the naked eye or brought out by etching of the surface. Depending on the temperature reached in the grinding zone a burn degree on the part surface can be observed which is due to temper color from very thin oxide layers on the part surface. This layer of ferrous material is composed of $\mathrm{Fe}_{2} \mathrm{O}_{3}, \mathrm{Fe}_{3} \mathrm{O}_{4}$, and $\mathrm{FeO}$ membranes 
from the free surface. At the onset of a grinding burn, the grinding force and the rate of wheel wear increase sharply, and the surface roughness deteriorates (Kwak \& Song, 2001; Badger \& Torrance, 2000). Other type of thermal damage is referred to as rehardening burn, which is caused by a metallurgical phase change in the material when the grinding temperature exceeds the austenizing temperature, creating a thin layer of hard, brittle, untempered martensite. To further exacerbate the problem, rehardening burn is also accompanied by secondary residual stress, because the newly formed material has a greater density than the original material (Badger \& Torrance, 2000)

In this study, the neural network has been applied to classifying the burn degrees obtained on the surfaces of the parts in grinding. The parameters of acoustic emission (AE), power signals and others derived from these signals have been used as the inputs of the neural network. The characterization of the surface quality of the ground parts was done by visual analyses with the naked eye and also by the software developed (Dotto, 2004).

What makes this work distinguished from others is the use of grinding parameters as input to the neural network, which have not been tested yet in burn classification by neural networks. Besides, a high sampling rate data acquisition system was employed to acquire the raw acoustic emission and cutting power.

\section{Grinding Burn Monitoring}

High temperatures generated in the grinding zone can cause several types of thermal damage to the part, for instance grinding burn in the case of steels, excessive tempering of the superficial layer with possible rehardening and increase of the brittleness, undesirable residual stress of tension, decrease of fatigue-life performance and micro-cracks. The decrease of grinding power is needed in order to minimize the restriction of thermal damages. This can be obtained by utilizing a softer grinding wheel or a rougher dressing operation but both present disadvantages (Malkin, 1989)

Grinding burn occurs during the cutting process when the amount of energy generated in the contact zone produces an increase of temperature enough to provoke a localized phase change in the material of the part. Such occurrence can be visually observed by the discoloration of the part surface (Malkin, 1989; Kwak \& Song, 2001; Kwak \& Ha, 2004). The burning is expected to occur when a critical temperature is exceeded in the grinding zone. He estimated a temperature rise of $720^{\circ} \mathrm{C}$ for burning to occur (Malkin, 1989).

Burn in steels is characterized by a visible bluish temper color on the ground surface. In steel, due to the burning phenomenon, the temper color changes from light brown to dark brown to violet to blue, in that order, depending on the severity of burn (Malkin, 1984; Nathan et al., 1999; Badger \& Torrance, 2000; Liu et al., 2005).

The root mean square value (RMS) of the acoustic emission signal has been the main parameter studied in previous grinding researches over a frequency band carefully selected. This signal has been a good parameter because it is rich in sound waves carrying a lot of useful information (Lee et al., 2006; Liu et al., 2006).

Aguiar et al. (2002) has demonstrated in their investigation that the combination of the acoustic emission (RMS) signal and the electric power signal of the electric motor that drives the wheel can provide meaningful parameters to indicate when grinding burn takes place. From the combination of these signals they obtained the parameter referred to as DPO for burn detection in grinding. This parameter consists of the relationship between the standard 
deviation of the RMS acoustic emission and the maximum value of the electric power in the grinding pass. The equation (1) describes the mentioned parameter.

$$
D P O=S_{E A} P_{\max }
$$

Where $\mathrm{S}_{\mathrm{EA}}$ is the standard deviation of the RMS acoustic emission; and $\mathrm{P}_{\max }$ is the maximum value of the electric power.

In the hope of more sensitivity to detect grinding burn, Dotto et al. (2006) has proposed a new parameter referred to as DPKS, which also utilizes the RMS acoustic emission and the power signals. This parameter is defined according to equation (2).

$$
D P K S=\left(\sum_{i=1}^{i=m}(P O T(i)-S(P O T))^{4}\right) \cdot S(E A)
$$

Where $i$ is the power index that varies from 1 to $m$ samples per grinding pass; $m$ is the number of samples of the pass; POT(i) is the instantaneous value of the power; $\mathrm{S}(\mathrm{POT})$ is the standard deviation of the power in the pass; S(EA) is the standard deviation of the RMS acoustic emission in the pass.

The statistics known as Constant False Alarm Rate (CFAR) and Mean Value Deviance (MVD) were employed successfully for detection of grinding burn (Wang et al. 2001; Aguiar et al., 2006b). The equation 3 represents the CFAR and the equation 4 the MVD.

$$
T_{c p l}(X)=\frac{\sum_{k=0}^{M-1} X_{k}^{v}}{\left(\sum_{k=0}^{M-1} X_{k}\right)^{v}}
$$

Where $X_{k}$ is the $k$-th is the magnitude-squared FFT bin, $v$ is a changeable exponent and $2 \mathrm{M}$ is the total number of FFT bins (due to conjugate symmetry, only half of the magnitudesquared FFT bins need be interrogated). Respectively $v=1$ and $v=\infty$ correspond to the energy detector and $\max \left\{X_{k}\right\} ; 2<v<3$ provides good performance in a wide range.

$$
T_{m v d}(X)=\frac{1}{M} \sum_{k=0}^{M-1} \log \left[\frac{\bar{X}}{X_{k}}\right]
$$

Where $\bar{X}_{\text {is the mean value of }}\left\{X_{k}\right\} ; \mathrm{M}$ and $X_{k}$ have the same meanings as in the CFAR statistic.

\section{Neural Network and its Application to Grinding}

Neural networks are composed of many non-linear computational elements operating in 
parallel fashion. Neural networks, because of their massive nature, can perform computations at a higher rate. Because of their adaptive nature in using the learning process, neural networks can adapt to changes in the data and learn the characteristics of input signals. Learning in a neural network means finding an appropriate set of weights that are connection strengths from the elements of this layer to the elements of a next layer (Kwak \& Ha, 2001).

There are three layers in a network, namely the input layer (which receives input from the outside world), the hidden layer (between the input and the output layers) and the output layer (the response given to the outside world) (Nathan et al. 1999). The neurons of different layers are interconnected through weights. Thus, a neural network is constituted by processing elements at different layers, interconnections between them, and the learning rules that define the way in which inputs are mapped on to the outputs. The usefulness of an ANN comes from its ability to respond to an input pattern in a desirable fashion, after the learning phase. As such, the processing units receive inputs and perform a weighted sum of its input values using the connection weights given initially by the user. This weighted sum is termed the activation value of the neuron, given by:

$$
u=\sum_{i=1}^{N} w_{i} x_{i}+\theta
$$

where $w_{i j}$ is the weight interconnecting two nodes $i$ and $j ; x_{i}$ is the input variable; and $u$ is the threshold value. During the forward pass through the network, each neuron evaluate an equation that expresses the output as a function of the inputs. Using the right kind of transfer function is therefore essential. A sigmoidal function can be used for this purpose, and is given by:

$$
f(x)=1 /\left(1+e^{-u}\right)
$$

Depending on the mismatch of the predicted output with the desired output, the weights are adjusted by back-propagation of error, so that the current mean square error (MSE) given by the following equation is reduced:

$$
M S E=\frac{1}{2 N K} \sum_{n=1}^{N} \sum_{k=1}^{K}\left(b_{n k}-S_{n k}\right)^{2}
$$

where $N$ is the number of patterns in the training data, $K$ is the number of nodes in the network, $b_{n k}$ is the target output for the $n$-th pattern and $s_{n k}$ is the actual output for the $n$-th pattern.

Still, it should be noted that the MSE itself is a function of the weights, as the computation of the output uses them. During this learning phase of the network the weights and the threshold values are adapted in order to develop the knowledge stored in the network. The weights are adjusted so as to obtain the desired output. The problem of finding the best set of weights in order to minimize the discrepancy between the desired and the actual 
response of the network is considered as a non-linear optimization problem (Nathan, 1999). The most popularly used learning algorithm, namely the back-propagation algorithm, uses an interactive gradient-descent heuristic approach to solve this problem. Once the learning process is completed, the final set of weight values is stored, this constituting the long term memory of the network, which is used later during the prediction process.

Previous investigations have proved the efficiency of the artificial neural networks in the prediction of grinding burn (Wang et al., 2005; Kwak \& Song, 2001; Kwak \& Ha, 2001; Nathan et al., 1999; Aguiar et al., 2005, Spadotto et al., 2006). Thus, this technique is very promising and can also be applied successfully to industrial automation in a flexible and integrated fashion.

\section{Methodology and Results}

A surface grinding machine from Sulmecânica manufacturer, Brazil, model RAPH-1055 was used in the grinding tests. The grinder was equipped with an aluminum oxide grinding wheel, from Norton Manufacturer, Model ART-FE-38A80PVH. A fixed acoustic emission sensor from Sensis manufacturer, model DM-42, placed near the workpiece and an electrical power transducer for measuring the electrical power consumed from the three-phase induction motor that drives the wheel were employed.

The workpieces for the grinding tests consisted of laminated bars of steel SAE 1020 ground in the shape of a prism with $150 \mathrm{~mm}$ length, $10 \mathrm{~mm}$ width and $60 \mathrm{~mm}$ height. The grinding process took place along the workpiece length.

The power transducer consists of a Hall sensor to measure the electric current and a Hall voltage sensor to measure the voltage at the electric motor terminals. Both signals are processed internally in the power transducer module by an integrated circuit, which delivers a voltage signal proportional to the electrical power consumed by the electric motor. The acoustic emission and the power signal are further sent to the data acquisition board from National Instrument, model PCI-6011, which is installed onto a personal computer. The LabVIEW software was utilized for acquiring the signals and storing them into binary files for further processing and analysis. The acoustic emission sensor used has a broad-band sensitivity of $1.0 \mathrm{MHz}$. Its amplifier also filtered the signal outside the range of $50 \mathrm{kHz}$ to $1.0 \mathrm{MHz}$. Figure 1 shows the schematic diagram of the grinding machine and instrumentation used.

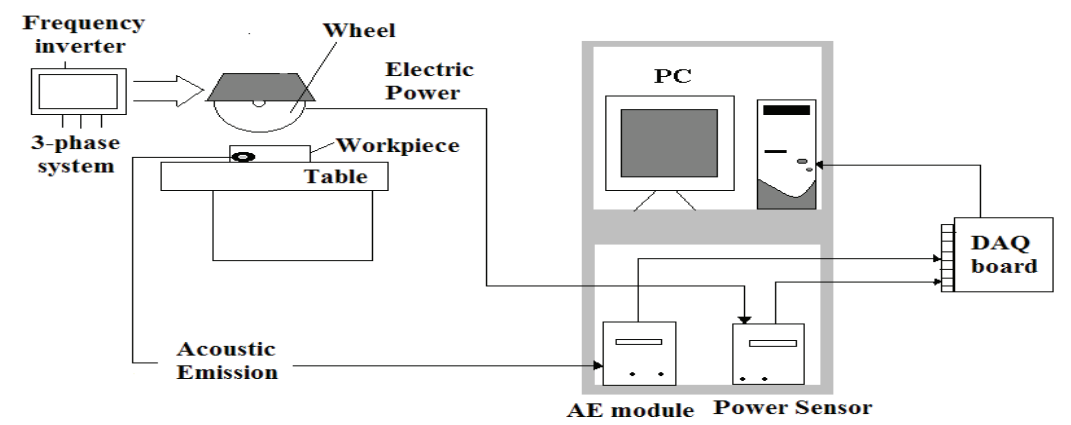

Fig. 1. Experimental setup. 
The tests were carried out for 12 different grinding conditions, and subsequently the burn degrees (no-burn, slight burn, medium burn, and severe burn) could be visually assessed for each workpiece surface. Dressing parameters, lubrication and peripheral wheel speed were adequately controlled in order to ensure the same grinding condition for each test. The workpiece speed was set up at $0.033 \mathrm{~m} / \mathrm{s}$ and the wheel speed at $30 \mathrm{~m} / \mathrm{s}$. The latter was maintained constant by adjusting the frequency of the induction motor on the frequency inverter, as the grinding wheel had its diameter decreased along the tests. The G-ratio, which is the volume of material removed per unit volume of wheel wear (Malkin, 1989), was set to 1, maintaining the dressing condition the same for all the tests. A water-based fluid was used with $4 \%$ concentration. Each run consisted of a single grinding pass of the grinding wheel along the workpiece length at a given grinding condition to be analyzed. The acoustic emission and cutting power signals were measured in real time at 2.0 millions of samples per second rate, and then stored onto binary data files for further processing. It is important to mention that the raw acoustic emission signal was acquired instead of the root mean square generally used.

The digital signal processing phase started after all the 12 tests were carried out and the data files stored. The digital signal processing of acoustic emission and power generated 7 new statistics as previously described, that is, the parameters DPO and DPKS, and the statistics CFAR and MVD. Seven structures were used for the neural network implementation as shown in Table 1. It can be noted in this table that besides the signals and statistics aforementioned the depth of cut a was also used as input of every structure.

\begin{tabular}{c|c|c|c}
\hline Structure & Inputs & Structure & Inputs \\
\hline I & Pot, AE, a & V & CFAR, a \\
\hline II & DPO, a & VI & AE, a \\
\hline III & DPKS, a & VII & Pot, a \\
\hline IV & MVD, a & & \\
\hline
\end{tabular}

Table 1. Neural network structures.

In this work, the back-propagation algorithm of neural networks, which is one of the learning models, was used. The following parameters were also found more suitable: downward gradient training algorithm; all data in the neural networks were normalized; training for 1000 epochs; square mean error value of 10-5. Cross-validation was used to estimate the generalization error of the model.

The outputs of the neural network was configured in a binary way according to the degree of burn obtained, that is, 0001 for no-burn, 0010 for slight burn, 0100 for medium burn, and 1000 for severe burn.

Each statistic was represented by a vector of 3000 samples for each test subsequently the digital processing of the acoustic emission and power signals. Initially, a visual analysis was carried out by naked eye on the part surfaces. Then, a quantification of the grinding burn on every part surface was done by a specific software for that purpose, which assessed the surface of a given part regarding the burn level through its digitalized picture (Dotto, 2004). Thus, a precise characterization of the burn levels occurred on the part surfaces was achieved. From the results of this characterization, input vectors were separated and 
assigned to the corresponding type of burn. The input vectors were again divided into training, validation and test vectors. Then, the process of optimization for the neural network was carried out.

The architecture of the neural networks was determined according to the tests of number of neurons of the hidden layer, learning rate and momentum. As the problem dealt in this work consists of pattern classification, only a simple hidden layer was chosen (Haykin, 1994). With learning rate equal to 1 and momentum fixed to zero the neurons of the hidden layer were varied at steps of 5 up to 50 neurons. The optimum number of neurons of the hidden layer was estimated according to the mean square error of the validation set of each structure calculated in the training phase. Figure 2 illustrates the comparison of errors obtained for each number of neurons considered for the structure 2 (DPO and depth of cut).

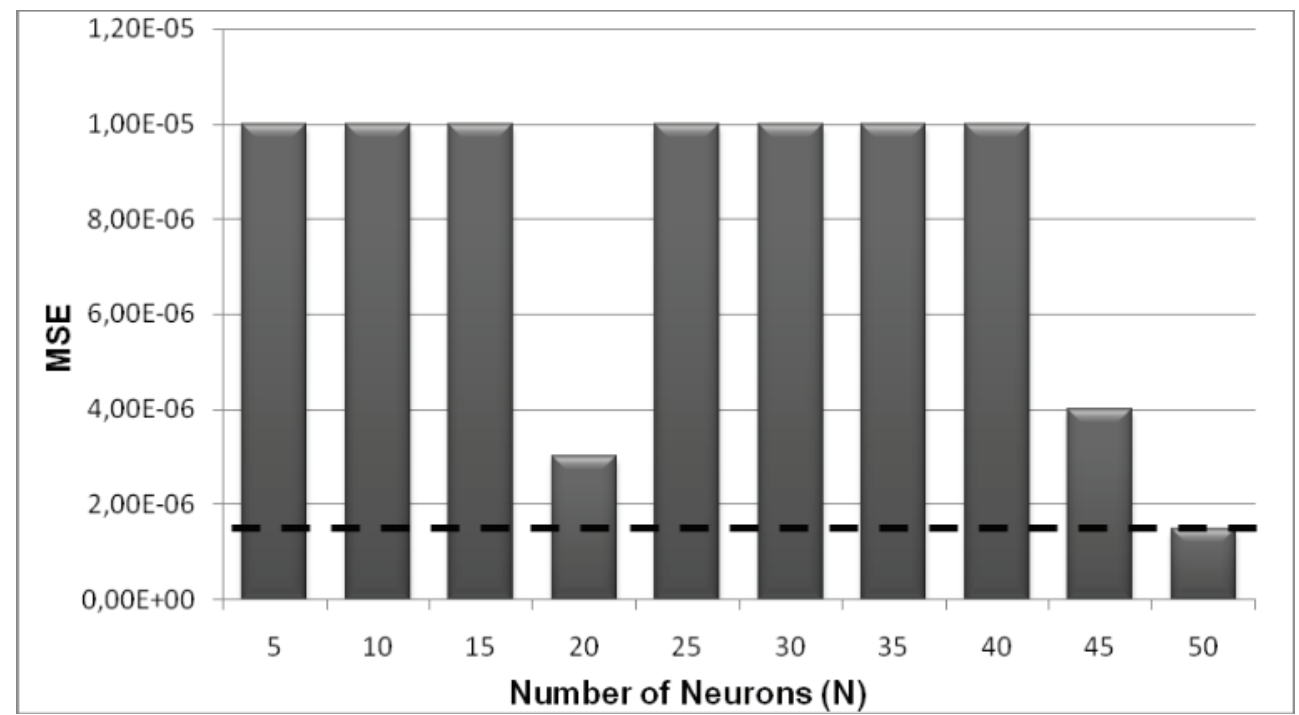

Fig. 2. Mean square error versus number of neurons of the hidden layer for structure 2.

In order to obtain the best values for learning rate and momentum the training was carried out for all aforementioned structures by varying these parameters. The proceeding for choosing the best pair momentum and learning rate was performed by fixing initially a value for momentum, varying the learning rate from 0.1 to 0.7 with step of 0.1 . After the error curves were obtained, the pair momentum and learning rate was chosen based on the curve which presented the smallest oscillation with the smallest number of epochs. This process was repeated for values of momentum from 0.2 to 0.7 , with step of 0.1 . Thus, the best six pairs were obtained in the end of this process, choosing among these pairs the one which presented the smallest oscillation with the smallest number of epochs.

Figure 3 illustrates the process of choice of the pair momentum and learning rate from comparisons between two curves of the mean square error in function of the number of epochs. These curves were obtained following the training of the structure 4 . 


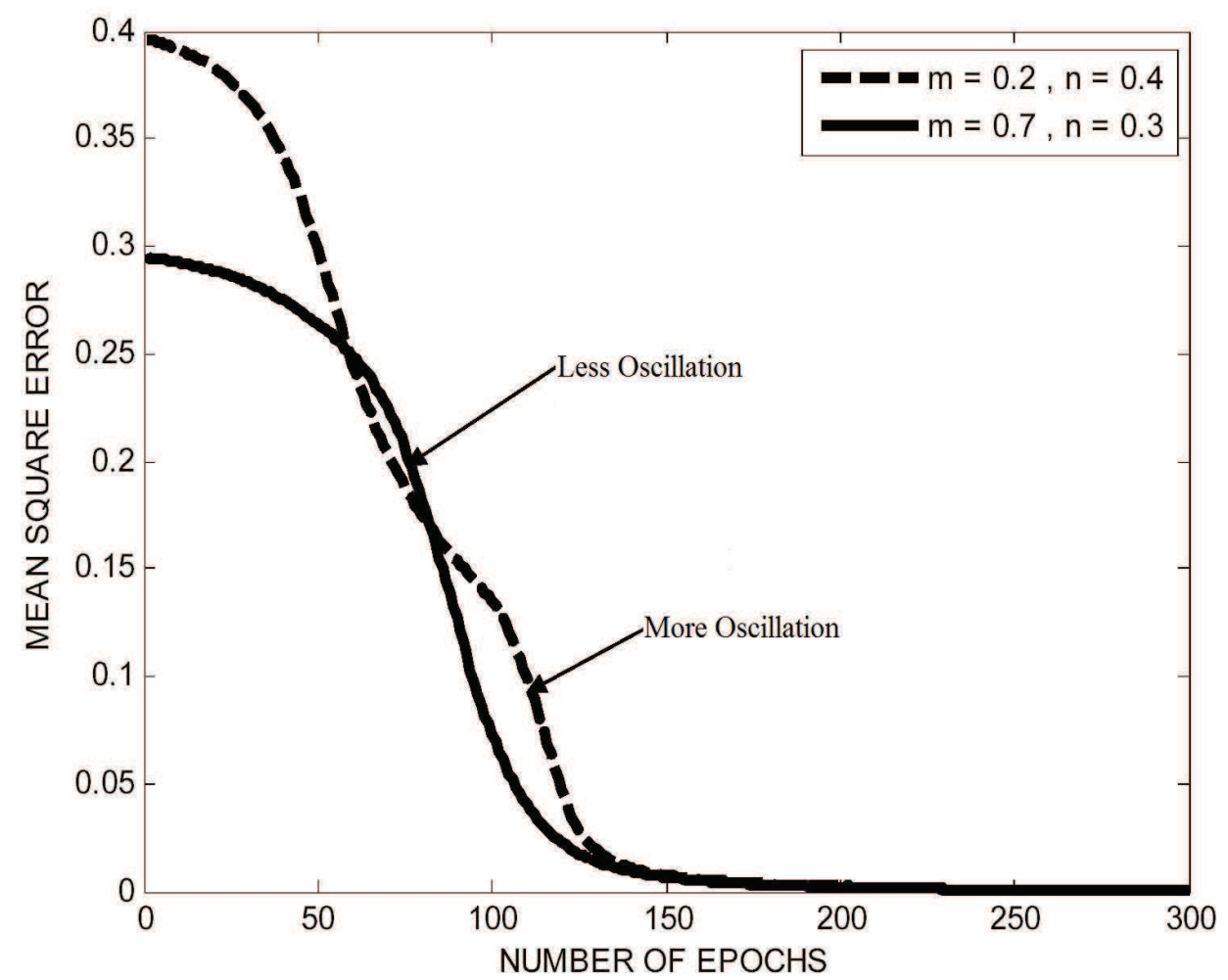

Fig. 3. Comparisons between two curves of the mean square error for a given pair momentum and learning rate for structure 4 .

It can be observed in Figure 3 two curves of mean square error in function of number of epochs showing different behaviours, that is, the curve with momentum 0.2 and learning rate 0.4 presented more oscillation than the curve with momentum 0.7 and learning rate 0.3 . Thus, the latter curve was chosen because it provided the smallest oscillation. This criterion is suggested by Haykin (1994) which states the learning rate and momentum lead to a local minimum in the error surface of the network with the smallest number of epochs.

On the other hand, the hidden neurons make the network to learn complex tasks by progressively extracting more significant characteristics of the input patterns (vectors). Moreover, because the change of the synaptic weight of the network is proportional to derivative of the activation function, it turns out that for the sigmoid activation function the synaptic weights are changed more intensely for those neurons of the network where the functional signal is in the middle of its interval. Based on these remarks, and also due to the previous experiences gotten from trial and error, the logsig activation function was chosen in this work for the neurons of the hidden and output layers.

On the basis of the optimization process of the neural network previously described in addition to the tests carried out for each structure, the best results for the all structures were obtained and presented in Table 2. 


\begin{tabular}{cccc}
\hline Structure & Neurons & Learning rate & momentum \\
\hline I & $3-35-4$ & 0.7 & 0.6 \\
II & $2-50-4$ & 0.7 & 0.3 \\
III & $2-45-4$ & 0.5 & 0.7 \\
IV & $2-30-4$ & 0.3 & 0.7 \\
V & $2-50-4$ & 0.7 & 0.3 \\
VI & $2-40-4$ & 0.7 & 0.7 \\
VII & $2-20-4$ & 0.5 & 0.3
\end{tabular}

Table 2. Configuration for the 7 neural network structures studied.

The neural networks of each structure were adjusted to have the correct parameters in the test phase for obtaining the number of neurons of the hidden layer, learning rate and momentum. The cross-validation method was employed with the training afterwards. The results for each structure were generated by inputting the corresponding data (signals and/or parameters) along with the depth of cut information, and the network output was interpreted in a bar graph fitting the form of the ground workpiece according to each burn level obtained. The digital picture of the workpiece with the corresponding bar graph for each structure was put all together for comparisons.

Figure 4 shows the results obtained when the signal vectors of test 2 not used in the training were inputted to the neural network. Thus, the data given to the neural network are different from those it was used to, testing this way its ability of classifying the burn levels.

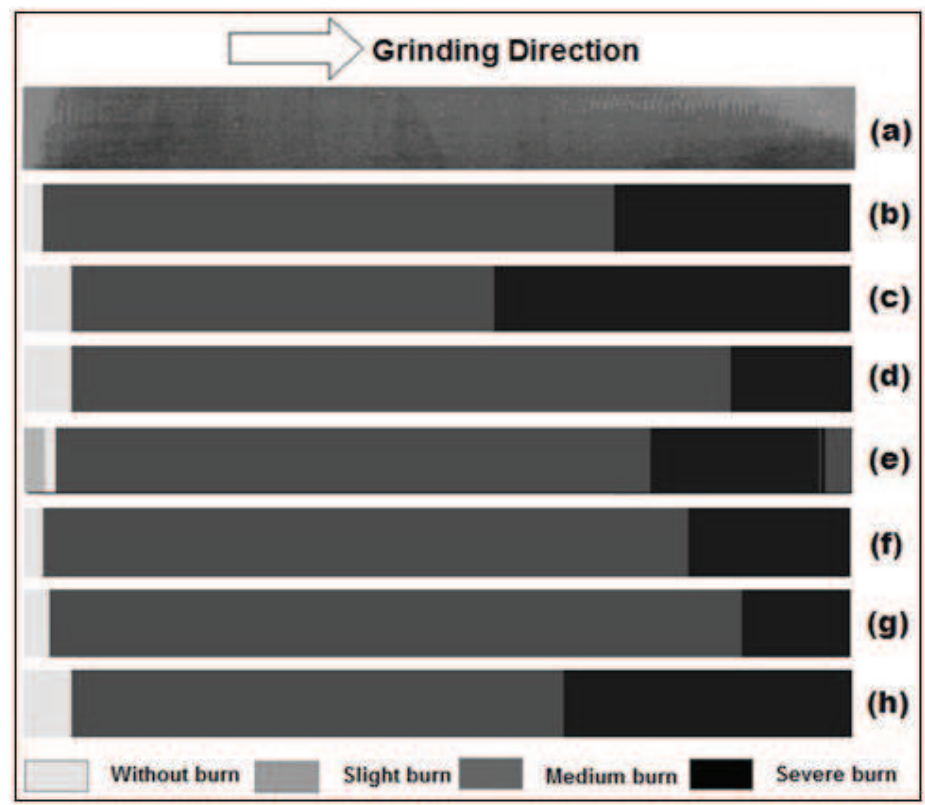

Fig. 4. Results obtained for Test 2; (a) Workpiece picture; (b) Structure 1; (c) Structure 2; (d) Structure 3; (e) Structure 4; (f) Structure 5; (g) Structure 6; (h) Structure 7. 
It can be observed in Figure 4 that the structures were able to detecting well the changes in the burn levels occurred in the test 2 . Some minor errors of classification were also observed as is the case of the structure 4, Figure 4(e), which has failed in classifying severe burn in the end of the workpiece, and the medium burn has been classified instead.

Following the classification performed for all structures, the percentage of success was calculated. Based on the comparisons of success percentages found for each structure, it was possible to determine the structure among all studied the one that presented more efficient at classifying the degrees of burn in the surface grinding process. Figure 5 shows the success rates in percentage illustrated by a bar graph as well as the grading of each structure. This ranking was carried out based on the general success rates for each structure, that is, considering the success of classifying workpieces with slight burn, medium burn, severe burn and no-burn.

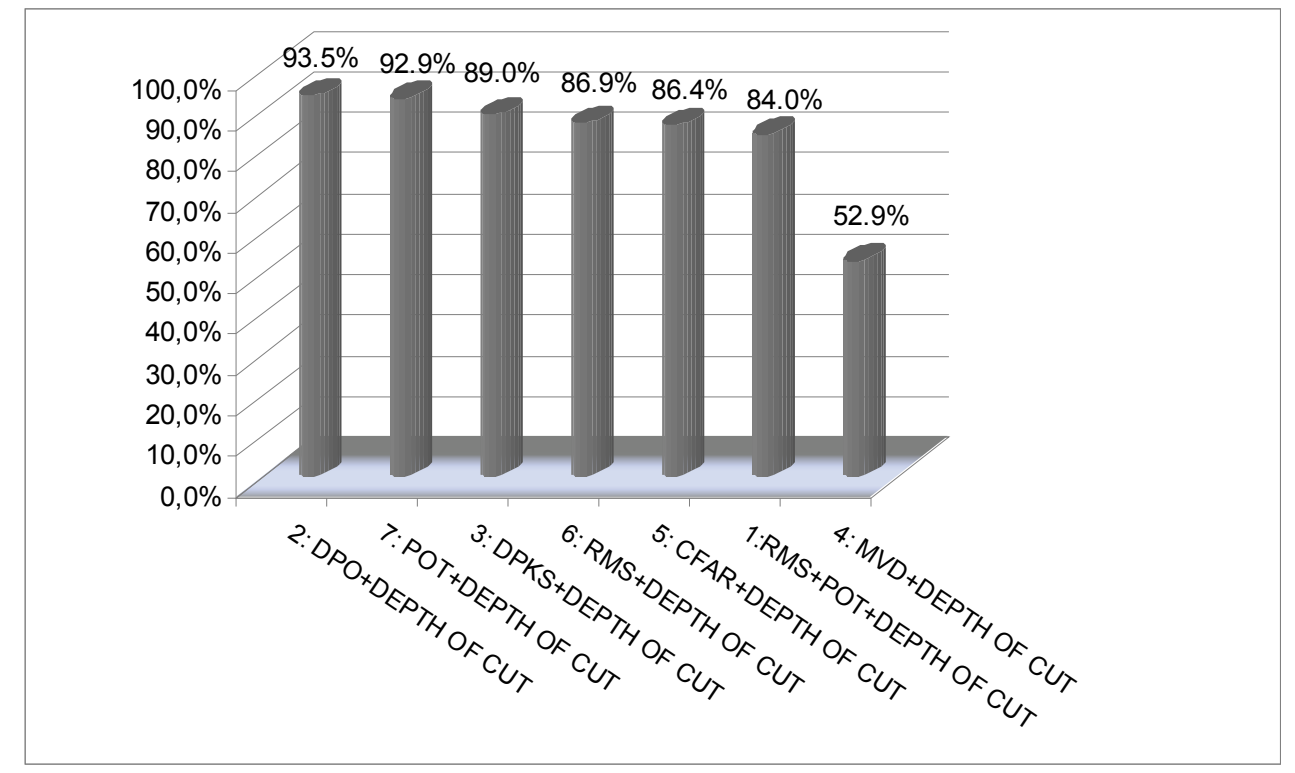

Fig. 5. Rate of success for each structure and the ranking obtained.

It can be observed in Figure 5 that all structures have presented a success rate quite good, with the exception of the structure 4 which has presented a success rate of only $52.9 \%$. The structure having acoustic emission, power and depth of cut was supposed to own a better position in the grading since these signals are widely employed in the grinding process monitoring. On the other hand, structure 2 composed by DPO parameter and depth of cut has presented the best result. This fact demonstrates the parameter DPO proposed by Aguiar et al. (2002) actually have a great sensitivity when thermal damage takes place in grinding, and therefore it shows up an excellent input to the neural network for solving classification problems of burn degrees. It can be emphasized that all structures detected slight burn quite well, and the grading showed in Figure 5 was built based on the success rate for all degrees of burn studied. 


\section{Conclusion}

The utilization of neural network of type multi-layer perceptron using the back-propagation algorithm guaranteed very good results. Tests carried out in order to optimize the learning capacity of neural networks were of utmost importance in the training phase, where the optimum values for the number of neurons of the hidden layer, learning rate and momentum for each structure were determined. Once the architecture of the neural network was established with those optimum values, the mean square error obtained during the training phase for the validation set proved that the neural network configuration was optimized, reaching values as lows as 1.0e-6. Moreover, the usage of cross-validation in the training phase was very important to right stop the training without overfitting occurrence, and then assuring a better generalization of the problem.

As all structures have detected correctly the degree of slight burn which is the first stage of change on thermal damage, it can be concluded that all structures worked well for classification of burn or non-burn occurrence.

The structure 2 with DPO and depth of cut as inputs presented the best results in comparison with the others studied. This can also be explained due to the parameter DPO combines the variations of the RMS acoustic emission and the maximum amplitude of the electric power during the grinding pass, resulting in an excellent tool for detection of burn degrees.

The differences of errors found among the structures 2, 6 and 7 are quite small, that is, less than $1 \%$ for the set of input \#7, and $6.6 \%$ for the set of input \#6 with respect to structure 2 . Therefore, the acoustic emission and electric power signals can also be employed successfully as inputs to the artificial neural networks for classification of burn degrees in grinding.

\section{Acknowledgments}

This work was supported by FAPESP -The State of Sao Paulo Research Foundation, IFM The Institute Factory of Millennium, and $\mathrm{CNPq}$ - National Council for Scientific and Technological Development (Grant \# 304842/2006-0). Also, thanks go to the Laboratory of Grinding and the Laboratory of Data Acquisition and Signal Processing at Unesp, campus Bauru, for the assets given to this work.

\section{References}

Aguiar, P. R.; Willett, P. \& Webster, J. (1998), Acoustic Emission Applied to Detect Workpiece Burn during Grinding. International Symposium on Acoustic Emission: Standards and Technology Update, S. Vahaviolos, Ed., Fort Lauderdale, FL. pp. 107124.

Aguiar, P. R.; Bianchi, E. C. \& Oliveira, J. F. G. (2002). A method for burning detection in grinding process using acoustic emission and effective electrical power signal, CIRP Journal of Manufacturing Systems, Vol. 31, Paris, 253-257.

Aguiar, P. R.; Bianchi, E. C.; Dotto, F. R. L.; Flauzino, R. A. \& Spatti, D. H. (2005). Neural network applied to detect burn in grinding. The IASTED International Conference on Artificial Intelligence and Applications, Innsbruck, Austria, 831-836. 
Aguiar, P. R.; França, T. V. \& Bianchi, E. C. (2006a). Roughness and roundness prediction in grinding, Proceedings of the 5th CIRP International Seminar on Intelligent Computation in Manufacturing Engineering (CIRP ICME '06), Italy, 183-188.

Aguiar, P. R.; Serni, P. J. A.; Dotto, F. R. L. \& Bianchi, E. C. (2006b). In-Process Grinding Monitoring Through Acoustic Emission. Journal of the Brazilian Society of Mechanical Sciences, Vol. XXVIII, No. 1, 118-124.

Badger, J. A. \& Torrance, A. (2000). Burn awareness - Understanding the causes of grinding burn helps alleviate the problem, Cutting Tool Engineering Magazine, Vol. 52, 12.

Dotto, F. R. L.; Aguiar, P. R.; Bianchi, E. C.; Serni, P. J. A. \& Thomazella, R. (2006). Automatic system for thermal damage detection in manufacturing process with internet monitoring, Journal of Brazilian Society of Mechanical Science $\mathcal{E}$ Engineering, Vol XXVIII, No. 2, 153-160.

Dotto, F. R. L. (2004). Online automatic detection system of thermal damages in the grinding process via Internet (in Portuguese). Master of Science Dissertation, Unesp, Bauru-SP. $220 \mathrm{p}$.

Haykin, S. (1994). Neural Networks - A Comprehensive Foundation, Macmillan, New York.

Kwak, J. S. \& Song, J. B. (2001). Trouble diagnosis of the grinding process by using acoustic emission signals, International Journal of Machine Tools \& Manufacture, Vol. 41, 899913.

Kwak, J. S. \& Ha, M. K. (2004). Neural network approach for diagnosis of grinding operation by acoustic emission and power signals, Journal of Materials Processing Technology, Vol. 147, 65-71.

Lee, D. E.; Hwang, I.; Valente, C. M. O.; Oliveira, J. F. G. \& Dornfeld, D. A. (2006). Precision manufacturing process monitoring with acoustic emission, International Journal of Machine Tools \& Manufacture, Vol. 46, 176-188.

Liu, Q.; Xun C. \& Gindy, N. (2005). Fuzzy pattern recognition of AE signals for grinding burn, International Journal of Machine Tools \& Manufacture, Vol. 45, 811-818.

Liu, Q.; Xun C. \& Gindy, N. (2006). Investigation of acoustic emission signals under a simulative environment of grinding burn, International Journal of Machine Tools $\mathcal{E}$ Manufacture, Vol. 46, 284-292.

Malkin, S. (1989). Grinding Technology - Theory and Applications of Machining with Abrasives, Ellis Horwood Limited Publishers, Chichester, Halsted Press: a division of John Wiley \& Sons.

Nathan, R. D.; Vijayaraghavan, L. \& Krishnamurthy, R. (1999). In-process monitoring of grinding burn in the cylindrical grinding of steel, Journal of Materials Processing Technology, Vol. 91, 37-42.

Spadotto, M. M. ; Aguiar, P. R.; Prazeres, R. C. ; Souza, C. C. P. \& Bianchi, E. C. (2006). Artificial neural network applied to detection of burning in grinding process. 5 th International Conference on Mechanics and Materials in Design, Porto, Portugal.

Wang, Z.; Willet, P.; Aguiar, P. R. \& Webster, J. (2001). Neural network detection of grinding burn from acoustic emission, International Journal of Machine Tools $\mathcal{E}$ Manufacture, Vol. 41, 283-309.

Wang, J. Z.; Wang, L. S.; Li, G. F.; \& Zhou, G. H. (2005). Prediction of surface roughness in cylindrical traverse grinding based on ALS algorithm. Proceedings of the Fourth Internacional Conference on Machine Learning and Cybernetics, Guangzhou, 549-554. 


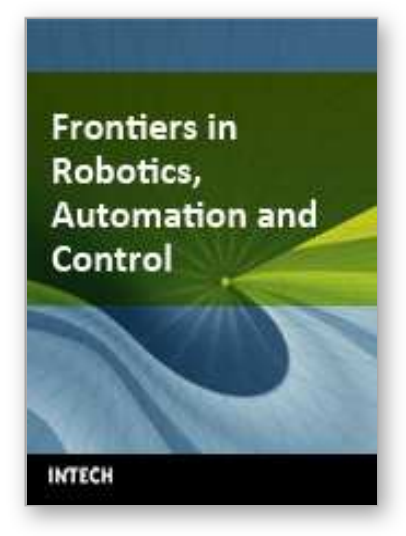

\author{
Frontiers in Robotics, Automation and Control \\ Edited by Alexander Zemliak
}

ISBN 978-953-7619-17-6

Hard cover, 450 pages

Publisher InTech

Published online 01, October, 2008

Published in print edition October, 2008

This book includes 23 chapters introducing basic research, advanced developments and applications. The book covers topics such us modeling and practical realization of robotic control for different applications, researching of the problems of stability and robustness, automation in algorithm and program developments with application in speech signal processing and linguistic research, system's applied control, computations, and control theory application in mechanics and electronics.

\title{
How to reference
}

In order to correctly reference this scholarly work, feel free to copy and paste the following:

Marcelo M. Spadotto, Paulo Roberto de Aguiar, Carlos C. P. Sousa and Eduardo C. Bianchi (2008). Neural Networks Applied to Thermal Damage Classification in Grinding Process, Frontiers in Robotics, Automation and Control, Alexander Zemliak (Ed.), ISBN: 978-953-7619-17-6, InTech, Available from:

http://www.intechopen.com/books/frontiers_in_robotics_automation_and_control/neural_networks_applied_to_ thermal_damage_classification_in_grinding_process

\section{INTECH}

open science | open minds

\section{InTech Europe}

University Campus STeP Ri

Slavka Krautzeka 83/A

51000 Rijeka, Croatia

Phone: +385 (51) 770447

Fax: +385 (51) 686166

www.intechopen.com

\section{InTech China}

Unit 405, Office Block, Hotel Equatorial Shanghai

No.65, Yan An Road (West), Shanghai, 200040, China

中国上海市延安西路65号上海国际贵都大饭店办公楼 405 单元

Phone: +86-21-62489820

Fax: $+86-21-62489821$ 
(C) 2008 The Author(s). Licensee IntechOpen. This chapter is distributed under the terms of the Creative Commons Attribution-NonCommercialShareAlike-3.0 License, which permits use, distribution and reproduction for non-commercial purposes, provided the original is properly cited and derivative works building on this content are distributed under the same license. 